\title{
SELF-CONSISTENT APPROXIMATIONS: APPLICATION TO A QUASIPARTICLE DESCRIPTION OF THE THERMODYNAMIC PROPERTIES OF RELATIVISTIC PLASMAS
}

\author{
Benoît Vanderheyden \\ The Niels Bohr Institute, Blegdamsvej 17, DK-2100 Copenhagen N, Denmark \\ Gordon Baym \\ Department of Physics, University of Illinois at Urbana-Champaign, \\ 1110 W. Green St., Urbana, Illinois 61801, USA
}

\begin{abstract}
We generalize the concept of conserving, $\Phi$-derivable, approximations to relativistic field theories. Treating the interaction field as a dynamical degree of freedom, we derive the thermodynamical potential in terms of fully dressed propagators, an approach which allows us to resolve the entropy of a relativistic plasma into contributions from its interacting elementary excitations. We illustrate the derivation for a hot QED plasma of massless particles. We also discuss how the self-consistency of the treatment manifests itself into relationships between the contributions from interaction and matter fields.
\end{abstract}

\section{INTRODUCTION}

Nuclear matter at very high densities is expected, because QCD is asymptotically free, to be in the form of a plasma of deconfined quarks and gluons. 1 Such plasmas were present in the early universe and may exist in the cores of neutrons stars; current experiments aim to produce and study them in collisions of ultrarelativistic nuclei. 2 In this talk, we address the question of providing a microscopic description of the thermodynamic properties of relativistic plasmas. The elementary excitations of weakly coupled quark-gluon plasmas are in fact, up to color factors, very similar in character to those of relativistic electromagnetic plasmas. Here, for simplicity we confine ourselves to the thermodynamic properties of QED plasmas. Unless otherwise stated, the properties of the electron and photon degrees of freedom which we consider can easily be translated into those of quark and gluon degrees of freedom in QCD plasmas.

Relativistic plasmas exhibit many of the familiar many-body effects encountered in non-relativistic condensed matter systems, including screening and the existence of collective modes. 3 Debye screening shields the static electric component of the interaction but does not affect static magnetic fields. Screening of finite frequency fields arises from Landau damping mechanisms in a manner similar to the anomalous skin effect in metals. 4 Early studies of relativistic plasmas have demonstrated the existence of bosonic and fermionic 
collective modes. 3 The bosonic excitations are the familiar longitudinal and transverse plasmon oscillations and appear as poles in the photon propagator. The fermionic collective excitations are unique to relativistic systems and develop as poles in the low momentum region of the electron propagator. Investigations of the fermionic spectrum at one-loop order have revealed the existence of a rich structure, including a gap at zero momentum and two distinct excitation branches at small momenta. 目

In the limit of small coupling constant $g$, these medium effects enter the theory through one-loop order corrections, which are systematically implemented by the "Hard-Thermal-Loop" (HTL) perturbation scheme of Braaten and Pisarski. $\mathrm{E}$ In a plasma of massless particles at temperature $T$, medium effects generically develop over the long wavelength scale $\sim 1 /(g T)$. (By comparison, the interparticle spacing is $\sim 1 / T$.) Difficulties, whose solution lies beyond the HTL scheme, arise from the lack of static screening of magnetic interactions. The long-ranged nature of static magnetic fields causes the fermion quasiparticle damping rate to be logarithmically divergent at finite temperature in perturbation theory. Blaizot and Iancu have shown that in high temperature QED inclusion of multiple scattering $\grave{a}$ $a$ Bloch-Nordsieck leads to well-defined, divergence-free quasiparticle modes. $\mathrm{C}$ In high temperature QCD, magnetic screening may actually appear at the scale $1 / g^{2} T$ due to gluon selfinteractions. The physics of the strong interactions at this scale is however non-perturbative and is thus again beyond the reach of HTL scheme. Related infrared difficulties due to long-ranged gauge fields arise in current issues of condensed matter theory, for instance in gauge field models of high $T_{c}$ superconductors and of the fractional quantum Hall effect.

In this talk, we present a general framework for analyzing the effects of the gap in the fermion spectrum and of the long-ranged gauge fields on the thermodynamic properties of relativistic plasmas. Since we are dealing with interacting degrees of freedom, care is needed to avoid overcounting. We generalize to relativistic systems the $\Phi$-derivable conserving approximations developed and Baym 9 (see also 10 ) in the context of quantum transport theories. As we shall see, this formulation permits the resolution of the entropy of a relativistic plasma into components relative to its elementary excitations. We will also show that the self-consistency of the approach implies subtle relationships between the contributions to the entropy from matter and interaction fields; these relationships are the manifestation of a proper counting of the degrees of freedom. 


\section{FROM THE THERMODYNAMICAL POTENTIAL TO THE ENTROPY}

We illustrate the derivation for a hot relativistic QED plasma, a gas of electrons and positrons in equilibrium with photons at temperature $T \gg m$, the electron mass. For simplicity, we set $m=0$. At finite temperature, the system is not Lorentz invariant; only rotational invariance is obeyed. There is thus a preferred frame - the rest frame of the system - in which medium effects act separately on the longitudinal and transverse components of the interaction. It is thus natural to choose the Coulomb gauge. We work in the imaginary time formalism.

As we have shown 12 the free energy $\Omega$ can be written in terms of the fully dressed electron and photon propagators $G$ and $D$ as

$$
\Omega / T=\Phi[G, D]-\operatorname{Tr} \Sigma G+\operatorname{Tr} \log \left(-\gamma^{0} G\right)+\frac{1}{2} \operatorname{Tr} \Pi D-\frac{1}{2} \operatorname{Tr} \log (-D) .
$$

The traces are over the four momenta and over spin and polarization states, so for instance

$$
\operatorname{Tr} \Sigma G \equiv \operatorname{tr} \sum_{p, n} \Sigma\left(\omega_{n}, p\right) G\left(\omega_{n}, p\right), \quad \operatorname{Tr} \Pi D \equiv \operatorname{tr} \sum_{q, n} \Pi\left(\omega_{n}, q\right) D\left(\omega_{n}, q\right),
$$

where $\omega_{n}=(2 n+1) i \pi T$ and $\omega_{n}=2 n i \pi T$ are the electron and photon Matsubara frequencies, and tr represents the traces over spins or polarizations. The second and third terms in Eq. (11) represent a contribution from the electron degrees of freedom and have a form similar to those encountered in nonrelativistic version of conserving approximations. The generalization to relativistic systems is implemented here by the last two terms, which correspond to treating the electromagnetic interaction as a dynamical degree of freedom. The coupling between electron and photon modes is described by the functional $\Phi[G, D]$, the sum of all two-particle-irreducible skeleton diagrams, expressed in terms of fully dressed $G$ and $D$ instead of the bare electron and photon propagators $G_{0}$ and $D_{0}$. Under a simultaneous variation of the propagators $G$ and $D$,

$$
\delta \Phi[G, D]=\operatorname{Tr} \Sigma \delta G-\frac{1}{2} \operatorname{Tr} \Pi \delta D,
$$

as one easily sees by removing an electron or photon line in a given diagram contributing to $\Phi$. The electron self-energy $\Sigma[G, D]$ and the polarization operator $\Pi[G, D]$ are here functionals of $G$ and $D$ and satisfy Dyson's equations

$$
G^{-1}=G_{0}^{-1}-\Sigma, \quad D^{-1}=D_{0}^{-1}-\Pi .
$$


We emphasize that the representation of the thermodynamical potential in Eq. (何) is exact. This result is based upon two essential properties of the $\Phi$ functional. The first given in Eq. (3), implies that the potential $\Omega$ is stationary under variations of $G$ and $D$ that do not modify the free propagators $G_{0}$ and $D_{0}$. The second property follows from the topology of the diagrams contributing to $\Phi$. As each vertex of a given $\Phi$-diagram is connected to two electron lines and one photon line, the functional $\Phi$ remains constant under the scaling transformations

$$
\Phi[G, D ; g]=\Phi\left[s^{-f} G, s^{2 f-2 v} D ; s^{v} g\right],
$$

where $g$ is the coupling constant at a vertex. To gain insight into the meaning of this property, we note that when combined with Eq. (3) it implies $\operatorname{Tr} \Sigma G=$ $-\operatorname{Tr} \Pi D$. This is one example of the interdependencies that arise between the electron and photon contributions to thermodynamic quantities when treated self-consistently. The traces $\operatorname{Tr} \Sigma G$ and $-\operatorname{Tr} \Pi D$ both represent the interaction energy between electrons and photons. These expressions are equal if $\Phi$ satisfies the scaling invariance of Eq. (5).

The principle behind the conserving approximations consists of choosing a physically motivated subset of diagrams contributing to $\Phi$, and deriving the corresponding self-energy functionals via Eq. (3). Dyson's equations then provide self-consistent equations for the electron and photon propagators. These approximations preserve the functional and topological properties of $\Phi$, and a proper counting of the degrees of freedom is ensured. In particular, it follows from the stationarity property of the thermodynamical potential $\Omega$ that the approximations obtained for the propagators $G$ and $D$ lead to current densities and an energy momentum tensor that obey the continuity equations for the conservation of charge, momentum, and energy 9 .

As we shall see shortly, the entropy $S=-\partial \Omega /\left.\partial T\right|_{\mu, V}$ is a quantity wellsuited to be analyzed in terms of its quasiparticle content. Converting the frequency sums into integrals in the usual way ${ }^{a}$, we write $S$ as

$$
S=-\left.\frac{\partial \Omega}{\partial T}\right|_{\mu, V}=S_{f}+S_{b}+S^{\prime}
$$

where

$S_{f} \equiv-\sum_{p \pm} \int_{-\infty}^{\infty} \frac{d \omega_{p}}{\pi} \frac{\partial f}{\partial T}\left(\operatorname{Im} \Sigma_{ \pm} \operatorname{Re} G_{ \pm}+\operatorname{Im} \log \left(-G_{ \pm}^{-1}\right)\right)$,

${ }^{a}$ Due to the zero Matsubara mode, the sum over photon frequencies becomes a principal value integral. 


$$
\begin{aligned}
S_{b} & \equiv-\sum_{q, l} f_{0}^{\infty} \frac{d \omega_{q}}{\pi} \frac{\partial n}{\partial T}\left(\operatorname{Im} \Pi_{l} \operatorname{Re} D_{l}+\operatorname{Im} \log \left(-D_{l}^{-1}\right)\right) \\
S^{\prime} & \equiv-\frac{\partial(T \Phi)}{\partial T}-\sum_{p \pm} \int_{-\infty}^{\infty} \frac{d \omega_{p}}{\pi} \frac{\partial f}{\partial T} \operatorname{Im} G_{ \pm} \operatorname{Re} \Sigma_{ \pm}-\sum_{q, l} f_{0}^{\infty} \frac{d \omega_{q}}{\pi} \frac{\partial n}{\partial T} \operatorname{Im} D_{l} \operatorname{Re} \Pi_{l}
\end{aligned}
$$

and where $f=\left(\exp \beta\left(\omega_{p}-\mu\right)+1\right)^{-1}$ and $n\left(\omega_{q}\right)=\left(\exp \beta \omega_{q}-1\right)^{-1}$ are Fermi and Bose occupation factors. The sum over internal degrees of freedom includes in $S_{f}$ a sum over states with helicity equal (+) and opposite to (-) their chirality, while it involves in $S_{b}$ a sum over the two transverse and the longitudinal polarizations. We note that in the expressions above only the temperature derivatives acting on occupation factors need to be retained. All other temperature dependences cancel out as a consequence of the stationarity of $\Omega .{ }^{12}$ The terms $S_{f}$ and $S_{b}$ represent the contributions from the electron and photon elementary modes. The term $S^{\prime}$, which describes residual interactions between quasiparticles, has the particular property that it vanishes at one-loop order, 12 corresponding to the $\Phi$-diagram and the self-energies in Fig. 1 . In the following, we will stay at this level of approximation and neglect $S^{\prime}$. An example of a higher-order interaction term $S^{\prime}$ appears in the description of the thermodynamic properties of ${ }^{3} \mathrm{He}$, where it describes the effects of repeated scattering of particle-hole pairs and gives rise to the $T^{3} \log T$ term in the low temperature specific heat. 13

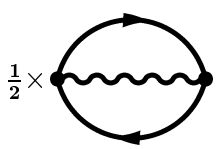

a)

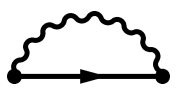

b)

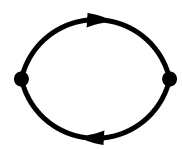

c)

Figure 1: (a) $\Phi$, (b) $\Sigma$ and (c) $\Pi$, to one-loop order.

\section{QUASIPARTICLE ANALYSIS OF THE ENTROPY OF AN ELECTROMAGNETIC PLASMA}

\subsection{A quasiparticle description of the thermodynamics of a QED plasma}

The reasons for choosing the entropy as the thermodynamic quantity to be analyzed in terms of its quasiparticle content are twofold. First, a simple inspec- 
tion of Eqs. (7)-(9) shows that, because of the presence of occupation factors in the integrand, the entropy does not suffer from ultraviolet divergences. Potential renormalization problems are thus avoided as all other thermodynamic quantities can be obtained from the entropy by simple integration. Second, a spectral representation can be introduced by recognizing that

$$
\frac{\partial f}{\partial T}=-\frac{\partial \sigma_{f}}{\partial \omega_{p}}\left(\omega_{p}\right), \quad \frac{\partial n}{\partial T}=-\frac{\partial \sigma_{n}}{\partial \omega_{q}}\left(\omega_{q}\right)
$$

where $\sigma_{f} \equiv-f \log f-(1-f) \log (1-f)$ and $\sigma_{n} \equiv-n \log n+(1+n) \log (1+n)$ are the entropy contributions from a single electron mode with energy $\omega_{p}$ and from a single photon mode with frequency $\omega_{q}$. Integrating Eqs. (7) and (8) by parts, we obtain the spectral representations

$$
S_{f}=\sum_{p} \int \frac{d \omega_{p}}{2 \pi} \sigma_{f}\left(\omega_{p}\right) A_{s}\left(\omega_{p}, p\right), S_{b}=\sum_{q} f_{0}^{\infty} \frac{d \omega_{q}}{2 \pi} \sigma_{b}\left(\omega_{q}\right) B_{s}\left(\omega_{q}, q\right)
$$

where the spectral density $A_{s}$ is defined as

$$
A_{s}\left(\omega_{p}, p\right)=\sum_{ \pm} \frac{\partial}{\partial \omega_{p}}\left\{\operatorname{Re} G_{ \pm} \operatorname{Im} \Sigma_{ \pm}+2 \operatorname{Im} \log \left(-G_{ \pm}\right)\right\},
$$

and $B_{s}$ has a similar structure in terms of $D_{l}$ and $\Pi_{l}$.

The physical content of the spectral densities can be elucidated as follows. For brevity, we only consider $A_{s}$. If the system develops well-defined excitations, the fermion propagator takes the following form near a quasiparticle pole:

$$
G_{ \pm}\left(\omega_{p}+i 0^{+}, p\right) \sim \frac{Z_{ \pm}}{\omega_{p}-\varepsilon_{p}^{ \pm}+i \Gamma_{p}^{ \pm} / 2}
$$

where $Z_{ \pm}$is the residue of the quasiparticle with energy $\varepsilon_{ \pm}$and inverse lifetime $\Gamma_{p}^{ \pm}=-2 \operatorname{Im} \Sigma_{ \pm}$. When the variation of $\Gamma_{ \pm}$around the quasiparticle pole is negligible, the density $A_{s}$ near the pole takes the form

$$
A_{s \pm}\left(\omega_{p}, p\right) \simeq \frac{\left(Z_{ \pm} \Gamma_{ \pm}\right)^{3} / 2}{\left(\left(\omega_{p}-\varepsilon_{p}^{ \pm}\right)^{2}+\left(Z_{ \pm} \Gamma_{ \pm} / 2\right)^{2}\right)^{2}} .
$$

Thus, in the non-interacting limit $\Gamma_{ \pm} \rightarrow 0, A_{s}$ reduces to a delta function and $S_{f}$ in Eq. (11) coincides with the entropy of a gas of free electrons with energies $\varepsilon_{p}^{ \pm}$. When $\Gamma_{ \pm}$is different from zero but small, so that well-defined quasiparticle modes exist, $A_{s}$ is a sharply peaked function which falls off faster 
than a Lorentzian. The excited quasiparticles as well as the degrees of freedom associated with their finite lifetimes thus contribute to the entropy $S_{f}$. The form of $B_{s}$ close to photon quasiparticles is qualitatively similar to that of $A_{s}$. Both $A_{s}$ and $B_{s}$ also have support over wide bands of continuum states, which describe the contributions from Landau damping effects. In higher order approximations, the continuum bands also include multiparticle states.

\subsection{Comparison to perturbative expansions}

The present $\Phi$ conserving approximations also provide a general framework for organizing calculations in perturbation theory. To illustrate we comment on the effects of the infrared structure and the presence of a gap in the fermion spectrum on the plasma thermodynamics. As mentioned earlier, the lack of static screening of magnetic fields is responsible for a logarithmic divergence of the damping rate evaluated in perturbation theory, $\Gamma_{ \pm}\left(\omega_{p}\right) \sim g^{2} T \log \left(g T / \mid \omega_{p}-\right.$ $\left.\varepsilon_{p}^{ \pm} \mid\right)$. This behavior implies that $A_{s}$ vanishes at the quasiparticle pole, since $A_{s} \sim 1 / \Gamma \sim 1 / \log \left(g T /\left|\omega_{p}-\varepsilon_{p}^{ \pm}\right|\right)$as $\omega_{p} \sim \varepsilon_{p}^{ \pm}$. There is thus no well-defined quasiparticles at this level of approximation; a correct quasiparticle description of the plasma thermodynamics must include a proper treatment of the longranged gauge fields for frequencies close to the poles $\varepsilon_{p}^{ \pm}$.

The effects of the gap in the fermion spectrum on thermodynamic quantities can also readily be estimated. Inspection of $S_{f}$ in Eq. (7) shows that low momentum modes with $\omega_{p} \sim p \sim g T$ contribute a factor $g^{5} T^{3}$ to the entropy per unit volume, in contrast with the contribution from the long-wavelength plasmon modes, $S_{\text {plasmons }} \sim g^{3} T^{3}$. 14 The difference in the order of magnitude can be traced back to statistics. For low frequencies $\omega_{p}, \omega_{q} \sim g T$, the temperature derivative $\partial f / \partial T \sim \omega_{p} / T^{2}$ in Eq. (7) is two powers of $g$ smaller than the derivative $\partial n / \partial T \sim 1 / \omega_{q}$ in Eq. (8). Hence $S_{f} \sim g^{2} S_{\text {plasmons }}$.

Further comparisons between our expressions and the standard results of perturbation theory reveal relations that follow from the self-consistent character of the derivation. For the formalism to reproduce all terms in the plasmon contribution to the entropy, $\sim g^{3} T^{3}$, one needs to include contributions from both long wavelength photons, via $S_{b}$, and electron modes, via $S_{f}$. These last terms describe the effects of the plasmons on the single particle energy of the electrons, via the real part of the self-energy, and are automatically included in a self-consistent treatment. The approach presented in this talk has recently been applied to purely gluonic QCD by Blaizot et al.,11 who have succeeded in obtaining an accurate quasiparticle description of lattice data for the equation of state down to temperatures twice $T_{c}$. There again, it is important to take into account the effects of soft modes on the dispersion relation of the hard 
modes to reproduce the entropy correctly up the order $g^{3}$. A description of these effects in terms of microscopic processes can be found in Ref. 11 .

\section{CONCLUSIONS}

In this talk, we generalized the concept of conserving approximations to relativistic theories. We have illustrated the technique for a hot electromagnetic plasma and shown how the approach allows one to resolve the entropy into contributions from the elementary degrees of freedom. We have also pointed out the advantages of implementing $\Phi$-conserving techniques as a basis for perturbation expansions.

\section{Acknowledgements}

This research was supported in part by NSF Grants PHY98-00978 and PHY9421309.

\section{References}

1. N. Itoh, Prog. Theor. Phys. 44, 291 (1970); P. Carruthers, Collective Phenomena 1, 147 (1973); J. C. Collins and M. J. Perry, Phys. Rev. Lett. 34, 1353 (1975); G. Baym and S. A. Chin, Phys. Lett. 62B, 241 (1976); M. Kislinger and P. Morley, Phys. Lett. 67B, 371 (1977).

2. See the proc. of the ongoing Quark Matter conferences, e.g., Nucl. Phys. A638 (1998).

3. For a review, see J. P. Blaizot, J.-Y. Ollitrault and E. Iancu in QuarkGluon Plasma 2, ed. R. C. Hwa (World Scientific, Singapore, 1996).

4. G.E.H. Reuter and E.H. Sondheimer, Proc. Roy. Soc. A195, 336 (1948).

5. V.V. Klimov, Sov. J. Nucl. Phys. 33, 934 (1981); H.A. Weldon, Phys. Rev. D26,2789 (1982); G. Baym, J.-P. Blaizot, and B. Svetitsky, Phys. Rev. D46, 4043 (1992); J.P. Blaizot and J.-Y. Ollitrault, Phys. Rev. D48, 1390 (1993).

6. E. Braaten and R.D. Pisarski, Phys. Rev. Lett. 64, 1338 (1990); Phys. Rev. D42, 2156 (1990); Nucl. Phys. B337, 569 (1990).

7. J.-P. Blaizot and E. Iancu, Phys. Rev. Lett. 76, 3080 (1996); Phys. Rev. D55, 973 (1997).

8. B.I. Halperin, P.A. Lee, and N. Read, Phys. Rev. B47, 7312 (1993);

L.B. Ioffe and A.I. Larkin, Phys. Rev. B39, 8988 (1989).

9. G. Baym, Phys. Rev. 127, 1391 (1962).

10. J. M. Luttinger and J. C. Ward, Phys. Rev. 118, 1417 (1960). 
11. J.-P. Blaizot, E. Iancu, and A. Rebhan, Phys. Rev. Lett. 83, 2906 (1999); hep-ph/9910309.

12. B. Vanderheyden and G. Baym, J. of Stat. Ph. 93, 843 (1998).

13. G. M. Carneiro and C. J. Pethick, Phys. Rev. A7, 304 (1973); Phys. Rev. B11, 1106 (1975), and refs. therein.

14. I. A. Akhiezer and S. V. Peletminskii, Sov. Phys. JETP 11, 1316 (1960). 